\title{
Measurements of cross sections for high energy neutron induced reactions on $\mathrm{Co}$ and $\mathrm{Bi}$
} \author{
Strugalska-Gola2 ${ }^{2}$, Mark Herbert ${ }^{7}$, and Vusumuzi Masondo ${ }^{7,8}$ \\ ${ }^{1}$ iThemba Laboratory for Accelerator Based Sciences, Faure, 7131, South Africa \\ ${ }^{2}$ National Centre for Nuclear Research, Otwock-Świerk 05-400, Poland \\ ${ }^{3}$ Joint Institute for Nuclear Research, 141980 Dubna, Russia \\ ${ }^{4}$ University of Cape Town, Rondebosch, Cape Town, 7700, South Africa \\ ${ }^{5}$ University of Zululand, KwaDlangezwa, 3886, South Africa \\ ${ }^{6}$ University of Fort Hare, Alice, 5700, South Africa \\ ${ }^{7}$ University of the Western Cape, Bellville, Cape Town, 7535, South Africa \\ ${ }^{8}$ Durban University of Technology, Greyville, Durban, 4001, South Africa
}

Ntombizikhona Ndlovu ${ }^{1}$, Peane Maleka ${ }^{1, *}$, Marcin Bielewicz ${ }^{2,3}$, Andy Buffler ${ }^{4}$, Frederick Smit $^{1}$, Dieter Geduld ${ }^{4}$, Sizwe Mhlongo $^{1,5}$, Thobeka Lamula ${ }^{1,5}$, Siyabulela Dyosi ${ }^{1,6}$, Rudolph Nchodu $^{1}$, Mathis Wiedeking ${ }^{1}$, Sifiso Ntshangase ${ }^{5}$, Elzbieta

\begin{abstract}
There are few experimental data for neutron cross-section libraries in $(n, x n)$ reactions for various materials at energies above $20 \mathrm{MeV}$. For neutron energies above $20 \mathrm{MeV}$, these set of (n,xn) reactions are important for neutron fluence monitoring and spectra unfolding for future generation IV nuclear reactors. There were attempts to measure the cross-sections on natural cobalt and bismuth at incident neutron energies of about $90 \mathrm{MeV}$ and $140 \mathrm{MeV}$. These measurements were made using the quasi-monoenergetic neutron facility at iThemba LABS, South Africa. In addition, at The Svedberg Laboratory facility in Sweden, similar experiments were performed on natural Yttrium. The measured cross-sections were compared with some of the few available data for neutron-induced reactions at high energies. Data collected from these two facilities, required corrections to be made for the contribution of the low energy tail (continuum) in the incident neutron spectrum. Preliminary results from iThemba LABS showed large discrepancy which we suspect was due to instability of the proton beam during the irradiations. Follow-up experiments are planned to accurately determine the uncertainty contributions, with additional data at other neutron energies.
\end{abstract}

\section{Introduction and Motivation}

At the iThemba LABS facility in South Africa, measurements of cross sections for the $(n, 3-6 n)$ reactions of various target materials using quasi mono-energetic neutron beams of 40 to $200 \mathrm{MeV}$ are ongoing. This campaign was initiated as part of the IRDFF (International Reactor Dosimetry and Fusion File) library, coordinated by the International atomic Energy Agency Nuclear Data Section (IAEA NDS). For example, the IRDF-2002 file that is available has experimental data for these dosimetry reactions which are insufficient, particularly at higher neutron energies, above the $20 \mathrm{MeV}$ threshold [1]. In addition, the few existing data have large uncertainties of about $30-50 \%$ and also show discrepancy between model predictions [1]. Figures 1 and 2 show respectively some of the available cross section data for the ${ }^{59} \mathrm{Co}(n, 3 n){ }^{57} \mathrm{Co}$ and ${ }^{209} \mathrm{Bi}(\mathrm{n}, 3 \mathrm{n})^{207} \mathrm{Bi}$ reactions. Experimental data for the ${ }^{59} \mathrm{Co}(\mathrm{n}, 3 \mathrm{n}){ }^{57} \mathrm{Co}$ reaction in fig. 1 , are based on experimental data collected in the seventies [2], then again in the late nineties [3, 4], followed by the more recent data [5]. Experimental data from Yashima (2017) is still

*e-mail: pmaleka@tlabs.ac.za to be verified by the IAEA-NDS [6]. In both figures 1 and 2 , the experimental data and the evaluated data agree in shape but not in absolute values. Moreover, for the ${ }^{209} \mathrm{Bi}(\mathrm{n}, 3 \mathrm{n}){ }^{207} \mathrm{Bi}$ reaction, there are no experimental data reported above $40 \mathrm{MeV}$ neutron energies.

At iThemba LABS, quasi-monoenergetic neutron beams are typically produced in the D-line experimental vault (refer to fig. 3) via the ${ }^{7} \mathrm{Li}(\mathrm{p}, \mathrm{n}){ }^{7} \mathrm{Be}$ or ${ }^{10} \mathrm{Be}(\mathrm{p}, \mathrm{n}){ }^{10} \mathrm{~B}$ reactions. Collimated fan beams are possible at various neutron emission angles, including $0^{\circ}$ and $16^{\circ}$. The peak is made up of neutrons emitted at $0^{\circ}$ from the ${ }^{7} \mathrm{Li}(\mathrm{p}, \mathrm{n})^{7} \mathrm{Be}$ reaction going to the ground and first excited states of ${ }^{7} \mathrm{Be}[7,8]$. The continuum is made up of neutrons from the breakup of ${ }^{7} \mathrm{Li}$, which is mainly isotropic up to an angle of $16^{\circ}$. Possible neutron flight paths extend from about 4 $\mathrm{m}$ to $10 \mathrm{~m}$. These neutron beams at iThemba LABS have been well characterized [8] in recent experiments, (see for example [9]) and methods for the accurate measurement of the spectral fluence have been developed [10]. By subtracting the yield produced in the $16^{\circ}$ beam line (after appropriate normalization) from that simultaneously 
produced at $0^{\circ}$ beam line, results in a yield determined for quasi-monoenergetic neutron energy, see [9] for more details.

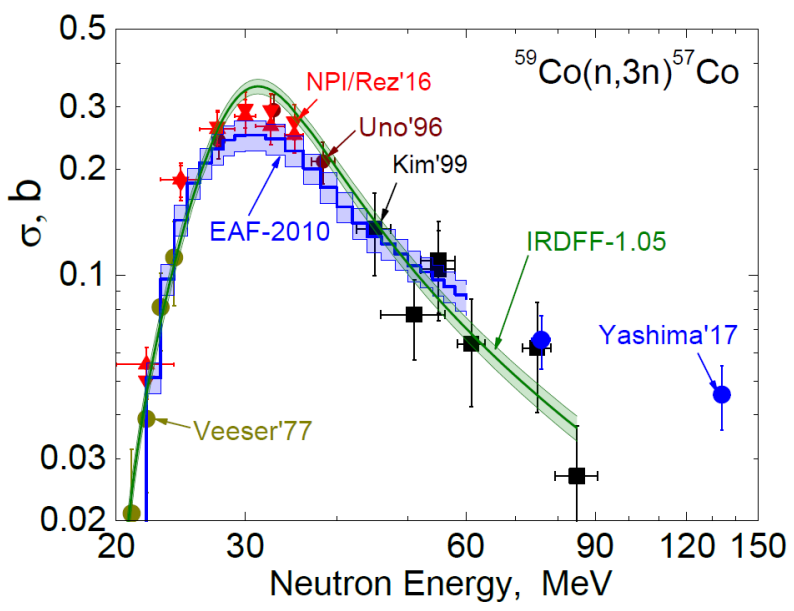

Figure 1. Compilation of experimental data and calculated neutron production cross-section of ${ }^{59} \mathrm{Co}(\mathrm{n}, 3 \mathrm{n}){ }^{57} \mathrm{Co}$ reaction in the energy range from $20 \mathrm{MeV}$ to $150 \mathrm{MeV}$ [6].

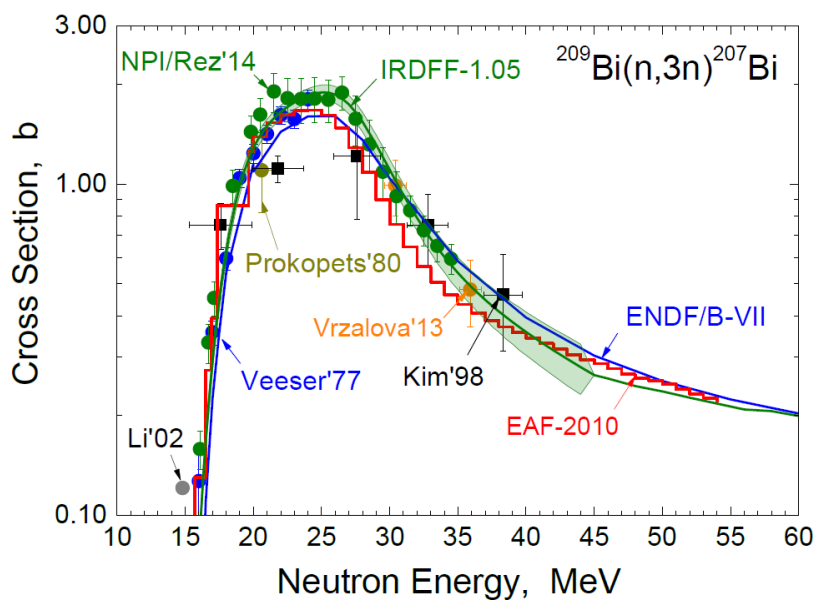

Figure 2. Compilation of experimental data and calculated neutron production cross-section of ${ }^{209} \mathrm{Bi}(\mathrm{n}, 3 \mathrm{n})^{209} \mathrm{Bi}$ reaction in the energy range from $15 \mathrm{MeV}$ to $100 \mathrm{MeV}$ [11].

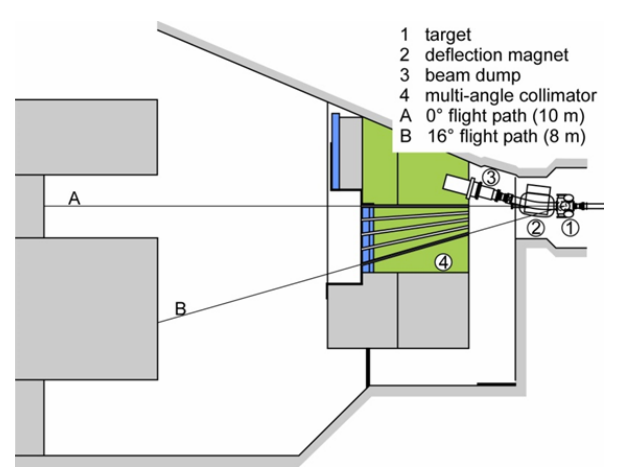

Figure 3. Schematic view of the iThemba LABS neutron beam facility (drawn not to scale).

The Svedberg Laboratory (TSL) facility, part of Uppsala University in Sweden, produced neutron beams up to $200 \mathrm{MeV}$. The quasi-monoenergetic neutron facility is located in the underground hall, referred to as the Blue Hall [12] (fig. 4). Neutrons were produced, by the accelerated proton beams going through a $4 \mathrm{~mm}$ or $23.5 \mathrm{~mm}$ thick ${ }^{7} \mathrm{Li}$ target. The neutrons, after passing the magnet area and the vacuum tubes, in state of a pure quasi-monochromatic neutron beam interact with foils/targets, located at $198 \mathrm{~cm}$ from the ${ }^{7} \mathrm{Li}$ target, and is called the Close User Position [13] (fig. 5). In the close user position, the beam intensity was much higher than in standard user position after collimator. This TSL facility capable of providing the quasi-monoenergetic neutrons was closed and this meant discontinuation of further cross-section measurement experiments.

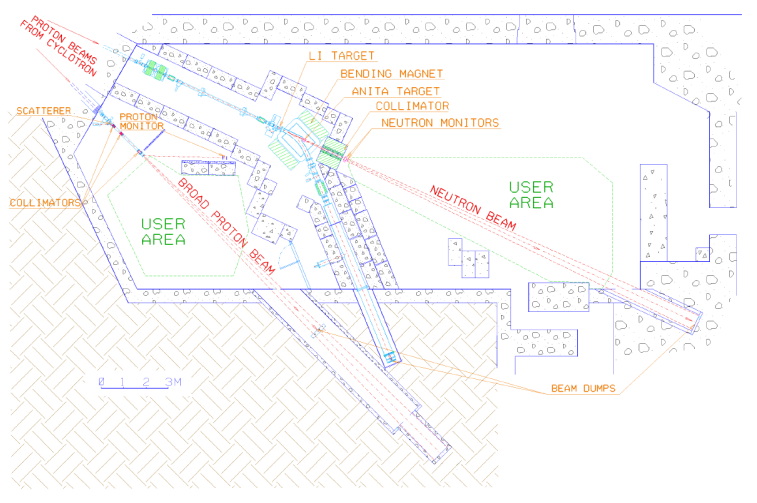

Figure 4. The Blue Hall scheme.

\section{Experimental Setup}

In 2014, experiments were performed at the iThemba LABS facility using the two peak neutron energies, about $90 \mathrm{MeV}$ and $140 \mathrm{MeV}$. Neutrons were produced by a proton beam accelerated from the cyclotron, to interact with a $8 \mathrm{~mm}$ thick ${ }^{7} \mathrm{Li}$ target. The proton beam is deflected by magnets into the beam dump after passing the target. Two identical target stacks (along the $0^{\circ}$ beam line and the other in the $16^{\circ}$ beamline (refer to figures 3 and 5)) 
that included ${ }^{59} \mathrm{Co}$ and ${ }^{209} \mathrm{Bi}$ materials were irradiated simultaneously for each neutron energy. Both irradiation positions were located about $5 \mathrm{~m}$ from the neutron production Li target and aligned using laser beams to the proton beam centre. In addition, $\mathrm{Al}$ and $\mathrm{Cu}$ targets are added in the stack as monitors (fig. 5). All target materials used were supplied by GoodFellow Corp. with $99.9 \%$ purity and as discs, $25 \mathrm{~mm}$ in diameter and $0.5 \mathrm{~mm}$ thick. The activated samples were thereafter counted using a low-background setup of gamma-ray detector, (hyper-pure germanium (HPGe), Canberra GC4520 model).

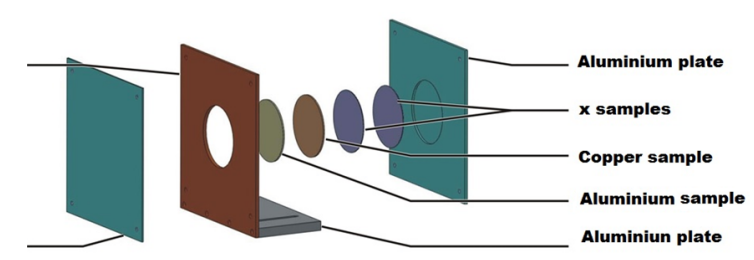

Figure 5. Typical target stack arrangement for the iThemba LABS irradiation experiments.

In 2015, experiments were performed at the quasimonoenergetic neutron facility [12] in TSL. The ${ }^{89} \mathrm{Y}$ foils were located at the close user position before metallic collimator (fig. 6) on the paperboard which was driven down using a special lift. The ${ }^{89} \mathrm{Y}$ foils were irradiated using four neutron peak energies, 35.5, 47.5, 60.5 and 92.5 MeV (see Table 1 for description of two of the energies).

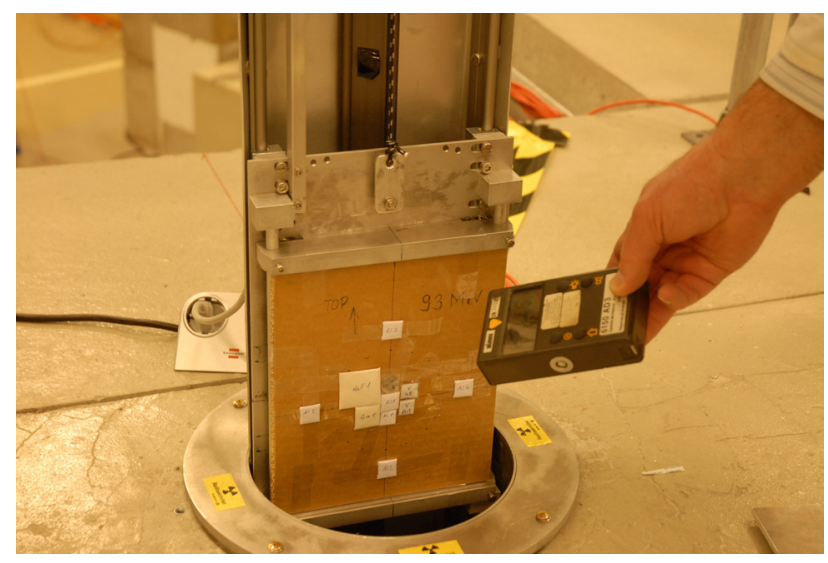

Figure 6. The samples/foils on the paperboard on the upper lift position (outside the bunker).

The description of calibration and calculation parameters, including the preliminary cross-section results for the two energies was reported by [14]. Final results of the four-neutron experiments still need to be reexamined in order to better identify the number of protons and value of background correction factor. The quasimonoenergetic neutron facility provides neutron beams with spectra comprising the high-energy peak and the lowenergy tail (fig. 7), with approximately equal fractions in the neutron spectrum. This feature of the neutron beams necessitates the second step in the data processing, namely the determination of the background correction factor. The background correction factor is defined as the ratio between the numbers of nuclei produced by the high-energy peak and by the whole neutron spectrum (high-energy peak plus low-energy tail) refer to [14].

Table 1. Beam and Calculation Parameters

\begin{tabular}{|l|l|l|}
\hline Proton Energy & $38 \mathrm{MeV}$ & $50 \mathrm{MeV}$ \\
\hline Neutron Energy Peak (calc.) & $35.5 \mathrm{MeV}$ & $47.5 \mathrm{MeV}$ \\
\hline Fraction of neutrons in the Peak (calc.) & $21.6 \%$ & $25.7 \%$ \\
${ }^{89} \mathrm{Y}(\mathrm{n}, 2 \mathrm{n}){ }^{88} \mathrm{Y}$ & $103(31) \mathrm{mbarn}$ & $95(28) \mathrm{mbarn}$ \\
\hline${ }^{89} \mathrm{Y}(\mathrm{n}, 3 \mathrm{n})^{87} \mathrm{Y}$ & $319(64) \mathrm{mbarn}$ & $198(40) \mathrm{mbarn}$ \\
\hline${ }^{89} \mathrm{Y}(\mathrm{n}, 4 \mathrm{n}){ }^{86} \mathrm{Y}$ & $338(68) \mathrm{mbarn}$ & $132(26) \mathrm{mbarn}$ \\
\hline
\end{tabular}

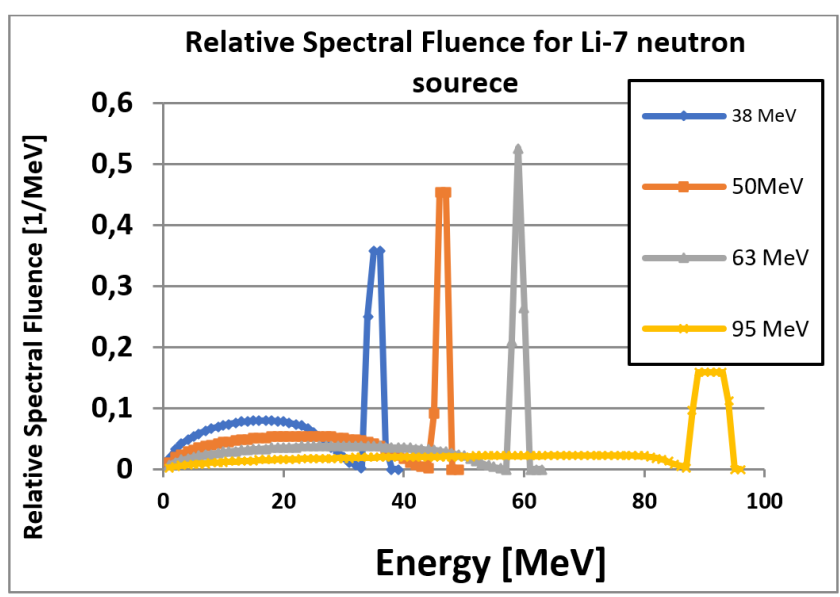

Figure 7. Relative spectral fluence of quasi-monochromatic neutrons with peak energies of $37.9,48.8,61.3$, and $95.5 \mathrm{MeV}$ at $0^{\circ}$ angle, calculated according to the algorithm from [15] and normalized so that the peak area is unity.

\section{Cross-section results and conclusion}

In this paper, we present status of analysis of the two target materials, cobalt and bismuth irradiated at the iThemba LABS facility. These preliminary results show discrepancy which we suspect was a result of proton beam instability during the irradiations.

The neutron spectra are shown in fig. 8, for the first experimental runs at $(90.0 \pm 3.6) \mathrm{MeV}$ and the follow-up runs at $(140.5 \pm 6.0) \mathrm{MeV}$. These data show some stability for the first runs, however the continuum in the follow-up experiments show some inconsistency with what was expected. In order to finalise the calculations for the crosssection values, corrections are required to be made for the contribution of the low energy tail in the incident neutron spectrum. In the previous study at iThemba LABS [9], it was estimated that about $7 \%$ uncertainty contribution come from the determination of the neutron fluences. With the observation in fig. 8, for these experiments we predict this contribution to be much higher than $20 \%$ for the $(90.0$ 
\pm 3.6) $\mathrm{MeV}$ and far worse for the $(140.5 \pm 6.0) \mathrm{MeV}$ neutron energy. The total uncertainty budget in these crosssection measurements will result from individual contributions; peak fluence determination, peak to continuum ratio, fluence monitor, HPGe detection efficiency and the counting statistics.
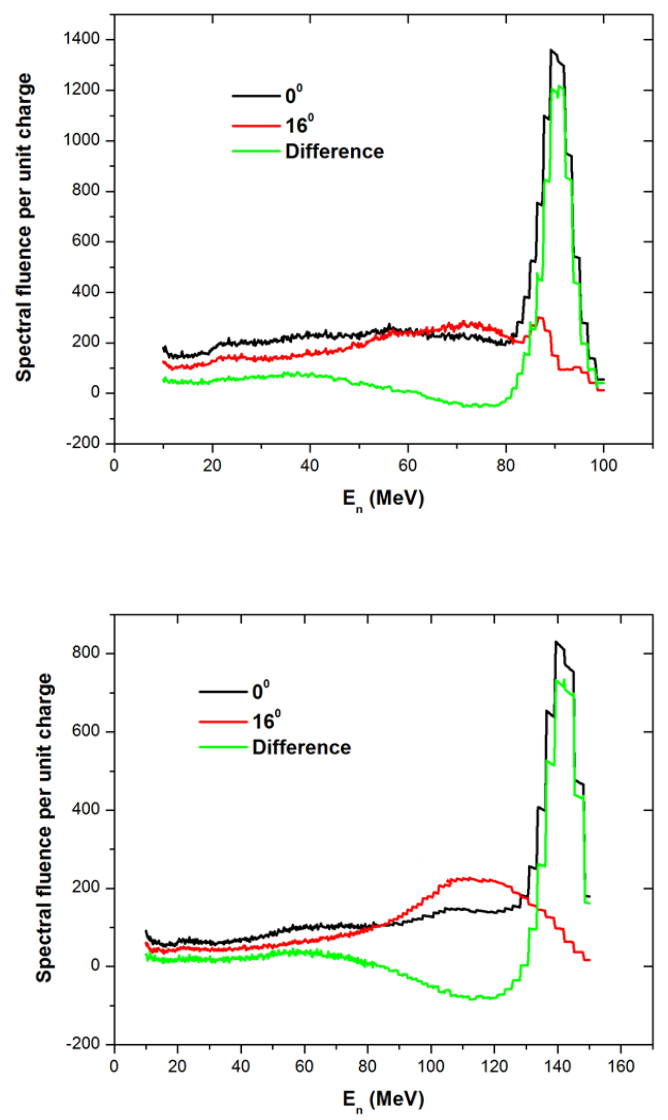

Figure 8. Spectral fluence of quasi-monoenergetic neutrons with peak energies of (top) $90 \mathrm{MeV}$ and (bottom) $140 \mathrm{MeV}$ at $0^{\circ}, 16^{\circ}$ angle and the normalized one using the difference method.

Gamma-ray spectra analysis are still ongoing to determine whether the information could be used to calculate the cross section despite the neutron spectra discrepancies. In conclusion, new irradiation initiatives are planned for the coming years and the approach is to include more neutron energies, between $30 \mathrm{MeV}$ and 200 $\mathrm{MeV}$.

\section{Acknowledgements}

This work at iThemba LABS is based on the research project supported by the National Research Foundation of South Africa and the Nuclear Data Section of IAEA, CRP number F41031. One of the authors (PM) would like to thank also both the NRF-iThemba LABS and the IAEA for providing funds to participate at the conference in China. We thank INIT and the accelerator departments for their assistance during the experiments at iThemba LABS.

\section{References}

[1] K.I. Zolotarev, IAEA, INDC(NDS)-0584 Report, (2010).

[2] L.R. Veeser et al., Physical Review C16, 1792-1802, (1977).

[3] E.J. Kim et al., Journal of Nuclear Science and Technology36, 29, (1999).

[4] Y Uno et al., Nuclear Science and Engineering 122, 247-257, (1996).

[5] M. Majerle et al., IAEA, INDC(CZR)-0002 Report, (2016).

[6] IAEA-NDS-Co: nds iaea org/IRDFFtest/Co(n,xn).pdf, July, 2019)

[7] Y. Iwamoto et al., Nuclear Instruments and Methods in Physics Research A 804, 50-58, (2015).

[8] R. Nolte et al., Nuclear Instruments and Methods in Physics Research A 476, 369-373, (2002).

[9] J M. Sisterson et al., Nuclear Instruments and Methods in Physics Research B, 240, 617-624, (2005).

[10] M. Mosconi et al., Radiation Measurement 45, 13421345, (2010).

[11] IAEA-NDS-Bi: nds.iaea.org/IRDFFtest/Bi(n,xn).pdf, July, 2019).

https://www-

(Accessed

[12] A.V. Prokofiev et al., Uppsala, Sweden; Rad. Prot. Dosim. v. 126, p.18-22 (2007)

[13] A. V. Prokofiev, et al, IEEE Transactions on Nuclear Science, v.61, p.1929-1936 (2014)

[14] M. Bielewicz et al., EPJ WoC 146, 11032 (2017)

[15] A.V. Prokofiev, et al.; Journal of Nuclear Science and Technology, Supplement 2, vol. 1, p.112-115 (2002) 